\title{
EFFECTS OF RETROGRADE CARDIOPLEGIA ON MYOCARDIAL PERFUSION AND ENERGY METABOLISM IN IMMATURE PORCINE MYOCARDIUM
}

Godwin Oriaku, MSca

Bo Xiang, DDS ${ }^{\mathrm{a}}$

Guangping Dai, $\mathrm{PhD}^{\mathrm{a}}$

Jie Shen, $\mathrm{PhD}^{\mathrm{b}}$

Jiankang Sun, MSc ${ }^{\mathrm{a}}$

William G. Lindsay, MD ${ }^{c}$

Roxanne Deslauriers, $\mathrm{PhD}^{\mathrm{a}}$

Ganghong Tian, MD, $\mathrm{PhD}^{\mathrm{a}}$
Objectives: Retrograde cardioplegia has been widely used for the protection of adult hearts during cardiac operations. Its efficacy to protect immature myocardium is still unclear. This study was designed to assess the effects of retrograde cardioplegia on myocardial perfusion and energy metabolism in immature hearts.

Methods: Piglet hearts were divided into 3 groups. Hearts in group 1 were used to assess myocardial perfusion of retrograde cardioplegia by means of magnetic resonance imaging. Hearts in groups 2 and 3 were used to assess the effects of retrograde cardioplegia on myocardial energy metabolism by use of phosphorus 31 magnetic resonance spectroscopy.

Results: Magnetic resonance images showed that perfusion with retrograde cardioplegic solution was heterogeneous. A perfusion defect was noted during retrograde cardioplegia in the right ventricular wall and in a portion of the posterior wall of the left ventricle in 4 of 6 hearts. Phosphorus 31 spectra showed that at the end of 45-minute retrograde cardioplegia, myocardial intracellular $\mathrm{pH}$ was $6.83 \pm 0.17$ and phosphocreatine was $53.5 \% \pm 27 \%$ of its prearrest value. The adenosine triphosphate level, however, remained normal throughout the retrograde cardioplegia period. Last, the hearts subjected to retrograde cardioplegia or antegrade cardioplegia showed similar and complete metabolic and functional recovery during reperfusion.

Conclusions: Retrograde cardioplegia provides heterogeneous perfusion. Its ability to protect the right ventricular myocardium is poor and varies between individuals. Myocardial perfusion provided by retrograde cardioplegia is slightly less than that needed to sustain normal myocardial energy metabolism under normothermic conditions. (J Thorac Cardiovasc Surg 2000;119:1102-9)
A ntegrade cardioplegia, hypothermia, and circulatory arrest have all been used in cardiac operations on neonates and infants. Hypothermia and antegrade cardioplegia have been the primary techniques used to minimize surgically induced myocardial ischemic injury. ${ }^{1-4}$ Because hypothermia cannot totally prevent

From the Institute for Biodiagnostics, National Research Council of Canada ${ }^{\mathrm{a}}$; Medical Systems, General Electronic, ${ }^{\mathrm{b}}$ Milwaukee, Wis; and the Department of Cardiovascular Surgery, University of Manitoba, Winnipeg, ${ }^{\mathrm{c}}$ Manitoba, Canada.

This work was supported by the Medical Research Council of Canada (grant 9609OP-348-BME-CDAA-26321) and the Heart and Stroke Foundation of Manitoba.

Received for publication Sept 8, 1999; revisions requested Nov 19, 1999; revisions received Jan 5, 2000; accepted for publication Feb 9, 2000.

Address for reprints: Ganghong Tian, 435 Ellice Ave, Winnipeg, Manitoba, Canada, R3B 1Y6 (E-mail: tian@ibd.nrc.ca).

12/1/106324

doi: $10.1067 / \mathrm{mtc} .2000 .106324$ myocardial ischemic injury, and delivery of antegrade cardioplegia may be compromised by some congenital deformations, there is still a need for further improvements in myocardial preservation strategies for cardiac operations on neonates and infants. 5,6

Retrograde perfusion of cardioplegic solution through the coronary sinus has been proven to be an effective technique for myocardial protection in adult hearts, especially during aortic valve operations and coronary artery bypass grafting. ${ }^{7-11}$ However, it has not been demonstrated whether this technique can provide adequate myocardial protection for neonatal and infant hearts. Because the venous system in immature myocardium may not develop as well as in mature myocardium, the efficacy of retrograde cardioplegia observed in adult hearts may not be seen in neonatal and infant hearts. The purpose of this study was to assess the effects of retrograde cardioplegia on myocardial perfusion and energy metabolism in immature 
hearts. Magnetic resonance (MR) imaging in combination with MR contrast agents was used to characterize the myocardial perfusion of retrograde cardioplegia. The ability of retrograde cardioplegia to sustain myocardial energy metabolism was studied by using phosphorous $31\left({ }^{31} \mathrm{P}\right) \mathrm{MR}$ spectroscopy. Antegrade cardioplegia was used as the control technique.

\section{Material and methods}

Isolated neonatal pig heart preparation. All animals used in this study received humane care in compliance with the "Guide to the Care and Use of Experimental Animals" formulated by the Canadian Council on Animal Care.

Eighteen domestic piglets aged 5 to 11 days and weighing 3.0 to $4.5 \mathrm{~kg}$ were sedated with an intramuscular administration of midazolam $(0.3 \mathrm{mg} / \mathrm{kg}$ body weight) and ketamine (20 $\mathrm{mg} / \mathrm{kg}$ body weight). Anesthesia was maintained with $1 \%$ to $2 \%$ isoflurane in a mixture of oxygen and nitrous oxide. The respiratory rate and volume were adjusted to keep the arterial blood gases within the normal physiologic range. A sternotomy was performed along the midline. Anticoagulation was provided by the injection of heparin (2000 IU) into the superior vena cava. Heparinized cold $\left(\sim 4^{\circ} \mathrm{C}\right)$ cardioplegic solution was infused into the aortic root $(20 \mathrm{~mL} / \mathrm{kg}$ body weight). The hearts were then excised and mounted on a Langendorff apparatus. A retrograde catheter with a manually inflated balloon at the tip $(6 \mathrm{~F}$; Medtronic of Canada Ltd, Mississauga, Ontario) was positioned into the coronary sinus in the hearts subjected to retrograde cardioplegia. The position of the catheter was adjusted so that the external edge of the balloon was at the orifice of the coronary sinus. The hemiazygos vein was ligated. The Langendorff system was then placed in the center of the magnet. The temperature of the hearts was maintained at $36.5^{\circ} \mathrm{C} \pm 0.5^{\circ} \mathrm{C}$ throughout the protocol. An in-line transonic flowmeter was used for monitoring the cardioplegic delivery rate.

Experimental groups. Piglet hearts were divided into 3 groups. The hearts in group $1(n=6)$ were used to assess the myocardial perfusion of antegrade cardioplegia and retrograde cardioplegia with MR perfusion imaging. All 6 hearts in this group were subjected to antegrade cardioplegia and retrograde cardioplegia at similar perfusion pressures (30-40 $\mathrm{mm} \mathrm{Hg}$ ) in a random order. An extracellular-type MR contrast agent (gadolinium diethylenetriamine pentaacetic acid [Gd-DTPA]; Berlex, Montreal, Quebec, Canada) was injected into the cardioplegic lines during antegrade cardioplegia and retrograde cardioplegia, respectively. The distribution of the contrast agent across myocardium was monitored with T1-weighted MR imaging. The perfusion medium containing the contrast agents was not recirculated, so that MR contrast agent injected during antegrade cardioplegia would have no effect on the subsequent retrograde cardioplegia. Complete washout of the contrast agent was also confirmed with MR imaging before subsequent contrast injection.

The piglet hearts in groups 2 and 3 were subjected to a protocol consisting of 30 minutes of control perfusion, 45 minutes of cardioplegic arrest, and 30 minutes of reperfusion. In the retrograde group $(n=6)$, cardioplegic arrest was induced with 2-minute antegrade cardioplegia and maintained with 43 -minute normothermic $\left(36.5^{\circ} \mathrm{C} \pm 0.5^{\circ} \mathrm{C}\right)$ retrograde cardioplegia (group $2, \mathrm{n}=6$ ). The hearts in group 3 $(n=6)$ were subjected to 45 minutes of continuous normothermic antegrade cardioplegia and used as controls. All piglets used in this study had different mothers, and they were assigned to the groups randomly. Myocardial energy metabolism and intracellular $\mathrm{pH}(\mathrm{pHi})$ were monitored throughout the protocol by ${ }^{31} \mathrm{P}$ MR spectroscopy. Myocardial contractile function was assessed during control perfusion and reperfusion periods.

Perfusion media. Because the amount of blood obtained from a piglet was not sufficient to prime the Langendorff system, the piglet hearts in this study were perfused with modified Krebs-Henseleit solution. During cardiac arrest, the piglet hearts were perfused with hyperkalemic KrebsHenseleit solution containing $100 \mathrm{mmol} / \mathrm{L} \mathrm{NaCl}, 16 \mathrm{mmol} / \mathrm{L}$ $\mathrm{KCl}, 16 \mathrm{mmol} / \mathrm{L} \mathrm{MgCl}_{2}, 0.5 \mathrm{mmol} / \mathrm{L}$ ethylenediamine tetraacetic acid, $11 \mathrm{mmol} / \mathrm{L}$ glucose, $25 \mathrm{mmol} / \mathrm{L} \mathrm{NaHCO}_{3}$, $1.75 \mathrm{mmol} / \mathrm{L} \mathrm{CaCl}_{2}, 1.2 \mathrm{mmol} / \mathrm{L} \mathrm{KH}_{2} \mathrm{PO}_{4}$, and $0.5 \%$ bovine serum albumin (Sigma Chemical Co, St Louis, Mo). During the control and reperfusion periods (for groups 2 and 3), the piglet hearts were perfused with normokalemic KrebsHenseleit solution containing $118 \mathrm{mmol} / \mathrm{L} \mathrm{NaCl}, 3.5 \mathrm{mmol} / \mathrm{L}$ $\mathrm{KCl}, 1.2 \mathrm{mmol} / \mathrm{L} \mathrm{MgSO}_{4}, 0.5 \mathrm{mmol} / \mathrm{L}$ ethylenediamine tetraacetic acid, $11 \mathrm{mmol} / \mathrm{L}$ glucose, $25 \mathrm{mmol} / \mathrm{L} \mathrm{NaHCO}_{3}$, $1.75 \mathrm{mmol} / \mathrm{L} \mathrm{CaCl}_{2}, 1.2 \mathrm{mmol} / \mathrm{L} \mathrm{KH}_{2} \mathrm{PO}_{4}$, and $0.5 \%$ bovine serum albumin. Both perfusion media were oxygenated with 95\% oxygen and $5 \%$ carbon dioxide to maintain its oxygen tension between 550 and $600 \mathrm{~mm} \mathrm{Hg}$ and $\mathrm{pH}$ at 7.35 to 7.45 . Perfusion pressure was controlled at 30 to $40 \mathrm{~mm} \mathrm{Hg}$ during cardiac arrest (retrograde cardioplegia and antegrade cardioplegia) and 70 to $80 \mathrm{~mm} \mathrm{Hg}$ during heart-beating periods, respectively.

Assessment of myocardial perfusion. Distribution of retrograde and antegrade cardioplegic solutions was determined by using T1-weighted MR imaging with Gd-DTPA. During retrograde cardioplegia and antegrade cardioplegia, $0.5 \mathrm{mmol}$ of Gd-DTPA was injected as a bolus into the perfusion line. During its first pass, the Gd-DTPA-induced T1 signal changes were measured by means of continuous acquisition of 200 T1-weighted images. Because Gd-DTPA increases the $\mathrm{T} 1$ signal intensities of the myocardium, bright areas in $\mathrm{T} 1$ images indicate the regions of perfused myocardium, whereas dark areas reveal nonperfused myocardium.

T1-weighted imaging was performed in a 7-T, 40-cm horizontal bore magnet equipped with a Biospec spectrometer (Bruker Inc, Karlsruhe, Germany) and a Helmholtz probe surrounding the whole heart. T1-weighted images were acquired by means of an inversion-recovery Turbo-FLASH sequence with a 3.6-ms echo time and a 6.4-ms repetition time. Each image covered a $12 \times 12 \mathrm{~cm}^{2}$ field of view with a $128 \times 128$ matrix, leading to a pixel size of $0.93 \times 0.93 \mathrm{~mm}^{2}$. All images were acquired from a $3-\mathrm{mm}$ slice parallel to the short cardiac axis. 

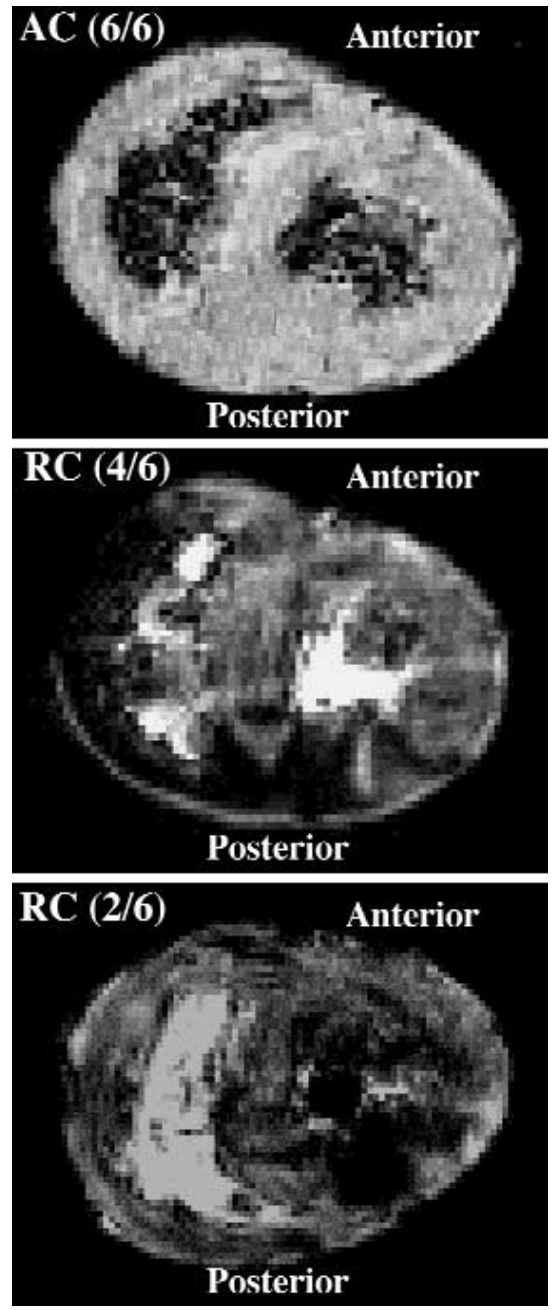

Fig 1. Representative T1-weighted MR images obtained during antegrade cardioplegia (AC, top panel) and retrograde cardioplegia ( $R C$, middle and bottom panels). Antegrade cardioplegia provided homogeneous perfusion across both ventricular walls. Retrograde cardioplegia did not provide cardioplegic solution to the RV wall in some hearts.

Study of the effects of retrograde cardioplegia and antegrade cardioplegia on myocardial energy metabolism. The effects of retrograde cardioplegia and antegrade cardioplegia on myocardial energy metabolism were studied in groups 2 and 3 by monitoring the levels of myocardial phosphocreatine $(\mathrm{PCr})$, inorganic phosphate $(\mathrm{Pi})$, adenosine triphosphate (ATP), and pHi by using ${ }^{31} \mathrm{P}$ MR spectroscopy. ${ }^{31} \mathrm{P}$ spectroscopy was performed on the magnet mentioned above, with a Helmholtz coil surrounding the whole heart.

Free induction decay signals were obtained by using 2048 data points, a 75-microsecond pulse length, and a 2.0-second repetition time. One hundred fifty free induction decay signals were accumulated for each spectrum over a 5-minute period. Intracellular $\mathrm{pH}$ was calculated from the chemical shift of the Pi peak relative to that of $\mathrm{PCr}{ }^{12,13}$ The $\beta$-peak of ATP was used for its quantification.

Assessment of contractile function. A latex balloon with its volume larger than that of the left ventricle (LV) was placed inside the LV to assess myocardial function of the hearts in groups 2 and 3. Myocardial contractile function was evaluated by measuring LV developed pressure (LVDP) and the maximal rate of $\mathrm{LV}$ pressure increase and decrease $( \pm \mathrm{dp} / \mathrm{dt})$. Contractile function was also evaluated by calculating the product of LVDP and heart rate to give the rate pressure product, an index of contractile work. Diastolic pressure of the LV was set at 0 to $5 \mathrm{~mm} \mathrm{Hg}$ during early period of control perfusion by inflating intraventricular balloon with water. The balloon volume achieved at the diastolic pressure was then kept unchanged throughout protocol.

Statistical analysis. Data are presented as means \pm SD. Statistical analyses were performed by using a statistical software STATISTICA (StatSoft, Inc, Tulsa, Okla). Comparisons of energy metabolites, $\mathrm{pHi}$, and cardiac contractile function between groups 2 and 3 were performed by analysis of variance with repeated measures. Significant changes in energy metabolites, $\mathrm{pHi}$, and contractile function that occurred throughout protocol within a group of animals were determined by analysis of variance.

\section{Results}

Effects of retrograde cardioplegia and antegrade cardioplegia on myocardial perfusion. Fig 1 shows representative MR images acquired with Gd-DTPA during antegrade cardioplegia (top panel) and retrograde cardioplegia (middle and bottom panels). As exemplified by the image in the top panel, all the images obtained during antegrade cardioplegia show a uniform distribution of MR contrast agent (6/6), suggesting homogeneous perfusion provided by antegrade cardioplegia. During retrograde cardioplegia, however, 4 of 6 hearts showed dark areas in the right ventricular (RV) free wall and in a small portion of the posterior wall of the LV (Fig 1, middle panel). This suggests that retrograde cardioplegia did not deliver cardioplegic solution to these regions of the myocardium in some hearts. In the remaining two hearts in the group, there was a similar increase in $\mathrm{T} 1$ signal in both ventricular walls during retrograde cardioplegia (Fig 1, bottom panel), suggesting that the ability of retrograde cardioplegia to protect the RV wall varies between each heart.

In addition, at similar perfusion pressures, retrograde cardioplegia provided significantly $(P<.05)$ less cardioplegic flow $(23.45 \pm 12.31 \mathrm{~mL} / \mathrm{min}$ per heart $)$ than that obtained with antegrade cardioplegia $(96 \pm 28.5$ $\mathrm{mL} / \mathrm{min}$ per heart).

Effect of retrograde cardioplegia and antegrade cardioplegia on myocardial energy metabolism. The changes in $\mathrm{PCr}, \mathrm{Pi}$, ATP, and pHi during retrograde car- 

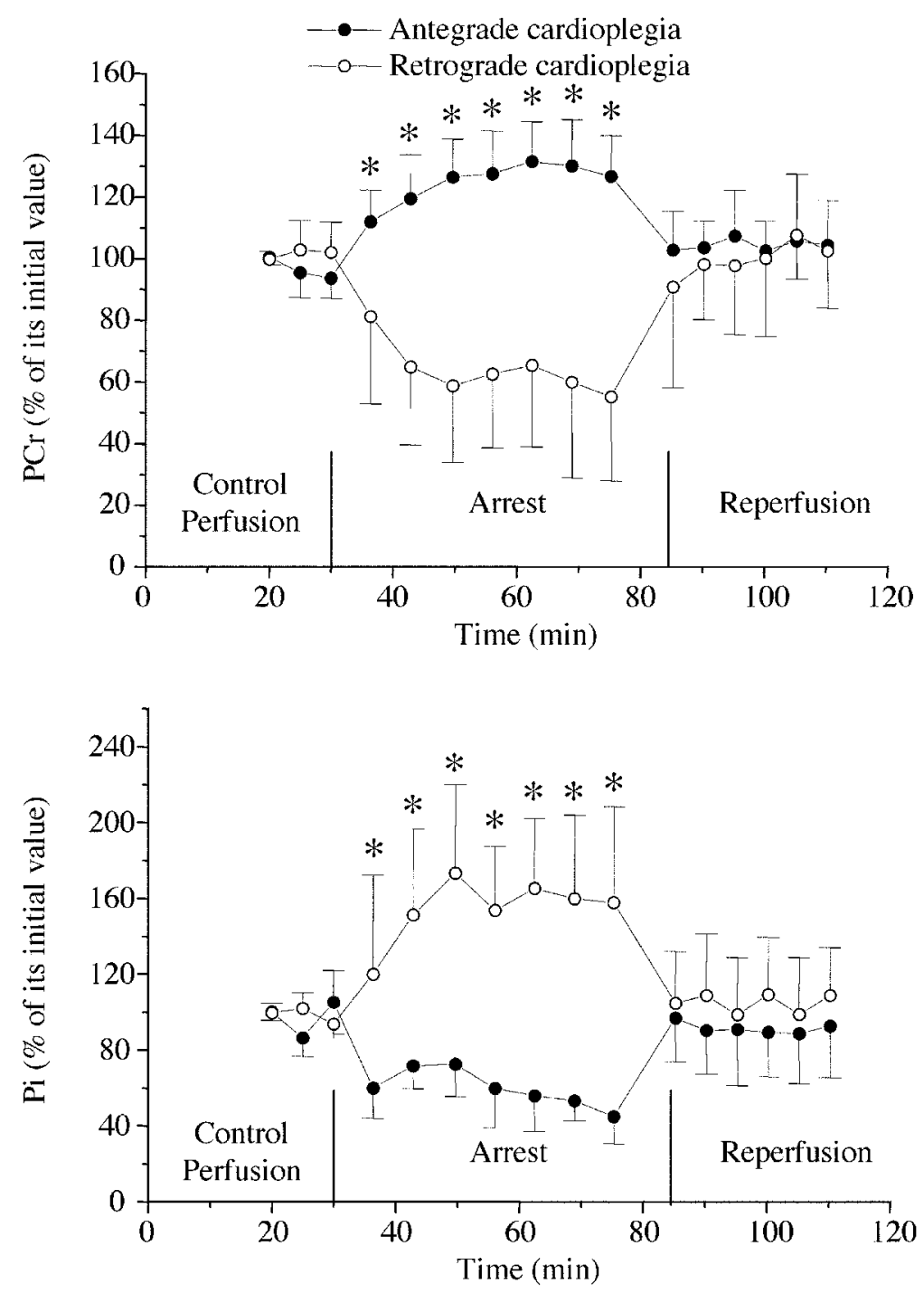

Fig 2. The changes in levels of phosphocreatine ( $P C r$, top panel) and inorganic phosphate ( $\mathrm{Pi}$, bottom panel) during control perfusion, cardiac arrest, and reperfusion. Asterisks indicate a significant difference between the two groups.

dioplegia and antegrade cardioplegia are shown in Figs 2 and 3. Retrograde cardioplegia resulted in a statistically significant $(P<.05)$ decrease in $\mathrm{PCr}$ and an increase in Pi levels. At the end of retrograde cardioplegia, the level of PCr was $53.5 \% \pm 27.3 \%$ of its control value obtained during control perfusion. Corresponding to the changes in $\mathrm{PCr}$ and $\mathrm{Pi}$, the hearts in the retrograde cardioplegia group showed a gradual decrease in pHi (Fig 3, bottom panel). Intracellular $\mathrm{pH}$ at the end of 43 minutes of retrograde cardioplegia $(6.83 \pm 0.17 \mathrm{pH}$ units $)$ was significantly lower than the control value (7.14 $\pm 0.10 \mathrm{pH}$ units) measured during control perfusion. These metabolic indices returned to normal soon after reperfusion. Retrograde cardioplegia did not result in any decline in ATP level (Fig 3, top panel). This suggests that the energy imbalance caused by retrograde cardioplegia was relatively mild. In contrast, antegrade cardioplegia resulted in a small but significant increase in PCr and a decrease in the Pi level. This suggests that antegrade cardioplegia at a perfusion pressure of 30 to $40 \mathrm{~mm} \mathrm{Hg}$ provides more than enough flow to sustain normal myocardial energy metabolism.

Effect of retrograde cardioplegia and antegrade cardioplegia on myocardial contractile function. Contractile function of the hearts in groups 2 and 3 during control perfusion and reperfusion is summarized in Fig 4. There is no significant difference in the rate pressure product (Fig 4, top panel) or $+\mathrm{dp} / \mathrm{dt}$ 

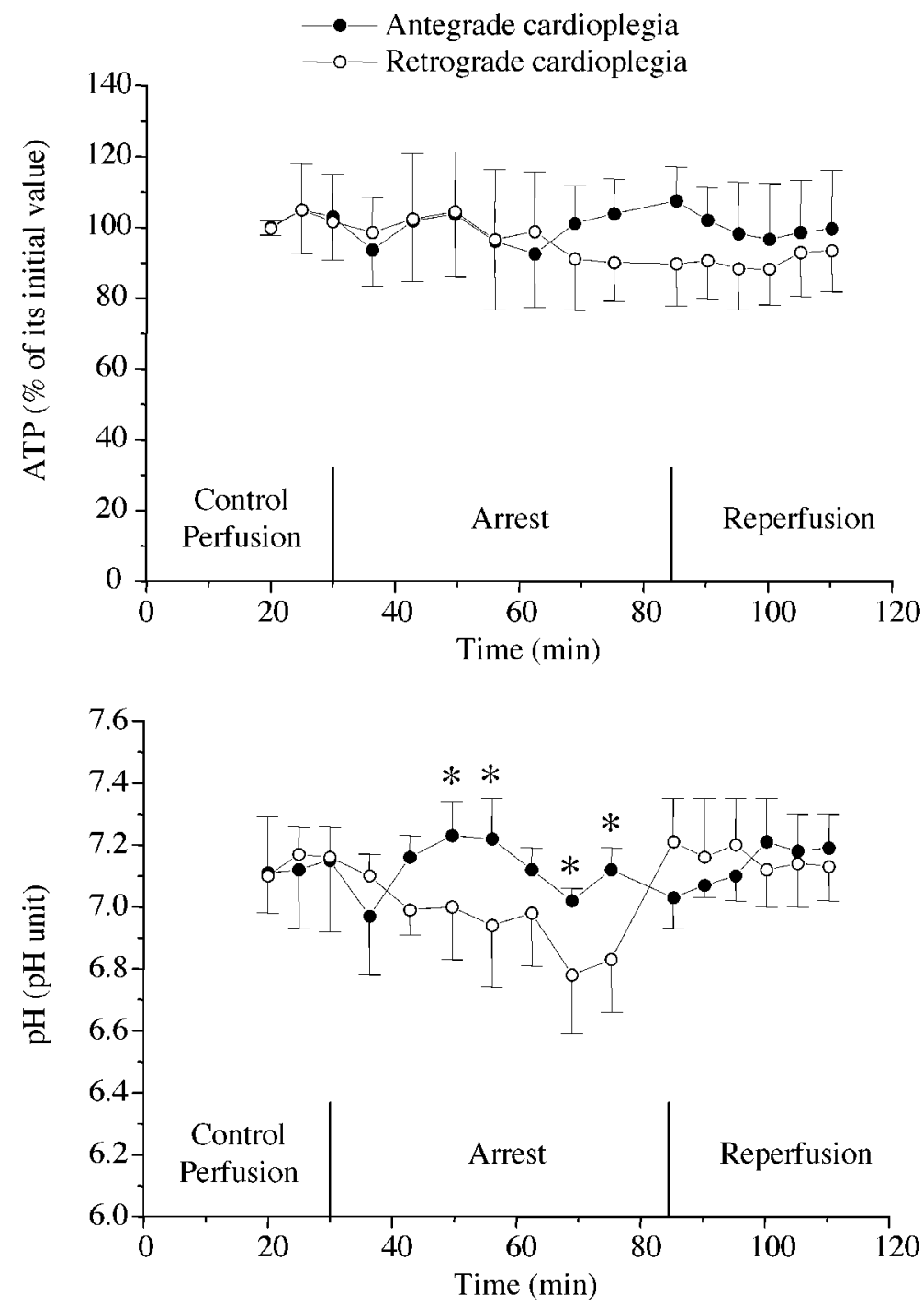

Fig 3. The time courses of changes in ATP (top panel) and intracellular $\mathrm{pH}$ (bottom). Asterisks indicate a significant difference between the two groups.

(Fig 4, bottom panel) between the two groups. Other measures of contractile function (LVDP and $-\mathrm{dp} / \mathrm{dt}$ ) were also comparable between the two groups (data not presented). Complete recovery of contractile function in the retrograde cardioplegia hearts during reperfusion further suggests that retrograde cardioplegia can provide sufficient myocardial protection in immature hearts, although it did not fully sustain normal myocardial energy metabolism.

\section{Discussion}

Retrograde cardioplegia has been widely accepted as a method of myocardial protection in adult heart operations. ${ }^{14-16}$ Its efficacy to protect immature hearts has not been demonstrated. The present study was designed to assess the effects of retrograde cardioplegia on myocardial perfusion and energy metabolism and to determine whether retrograde cardioplegia can provide adequate myocardial protection for immature hearts. MR images show that retrograde cardioplegia does not provide homogeneous perfusion across the myocardium. The ability of retrograde cardioplegia to perfuse the RV free wall varied significantly between individual hearts. Myocardial perfusion provided by retrograde cardioplegia was slightly less than that needed to sustain normal myocardial energy metabolism under normothermic conditions. However, the use of retrograde cardioplegia still resulted in complete recovery 

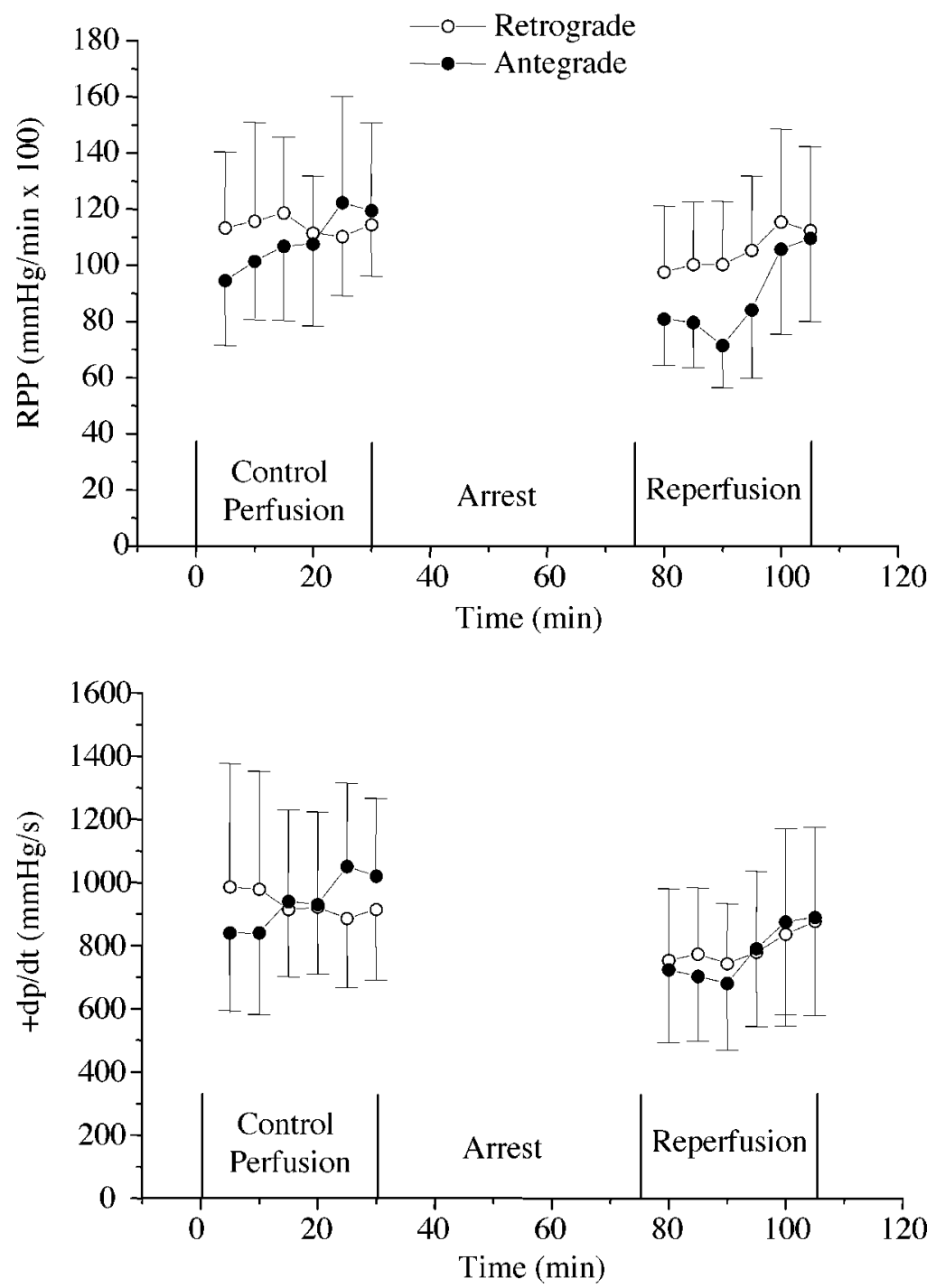

Fig 4. Comparison of rate pressure product (RPP, top panel) and the rate of $\mathrm{LV}$ pressure increase $(+d p / d t$, bottom panel) between the hearts subjected to antegrade cardioplegia and those subjected to retrograde cardioplegia.

of energy metabolism and LV contractile function during reperfusion.

During retrograde cardioplegia, T1-weighted images showed dark regions in the RV free walls of 4 of the 6 hearts (Fig 1, middle panel), indicating a lack of uniform delivery of cardioplegic solution to the RV free wall in some immature hearts. It has been suggested that the inability of retrograde cardioplegia to perfuse the RV myocardium is related to the diversion of cardioplegic solution from the venulae and capillaries by the thebesian vessels. ${ }^{17-20}$ This suggestion is made on the basis of the findings that under physiologic conditions the thebesian vessels help drain a considerable amount of venous blood into the RV chamber. ${ }^{17}$ The time course of $\mathrm{T} 1$ signal intensities obtained during retrograde cardioplegia did not show any transient increase of T1 signal in the RV wall (data not presented), suggesting that no contrast agent reached the RV wall. Therefore, the inability of retrograde cardioplegia to protect the RV wall is not due to thebesian drainage. We believe that blockage of the ostia of the coronary veins by the retrograde balloon and opening of the coronary veins directly to the right atrium are responsible for the lack of perfusion of the RV during retrograde cardioplegia. ${ }^{21,22}$ Two hearts in this group showed similar increases in T1 signal intensities in the 
$\mathrm{RV}(38.6 \pm 15.3 \mathrm{AU})$ and $\mathrm{LV}(42.3 \pm 37.15 \mathrm{AU})$ walls during retrograde cardioplegia, indicating that the ability of retrograde cardioplegia to protect the RV myocardium may depend on the individual heart because of the variation in coronary venous anatomy.

To assess the metabolic effects of retrograde cardioplegia, ${ }^{31} \mathrm{P}$ MR spectroscopy was used to follow myocardial energy metabolism. We found that $43 \mathrm{~min}-$ utes of normothermic retrograde cardioplegia resulted in a decrease in $\mathrm{PCr}$ and $\mathrm{pHi}$ and an increase in $\mathrm{Pi}$, suggesting that the level of myocardial perfusion provided by retrograde cardioplegia was less than that required to sustain myocardial energy metabolism under normothermic conditions.

On the basis of the levels of high-energy phosphates, reversible myocardial ischemia can be arbitrarily divided into 3 stages: latency, survival time, and revival time. ${ }^{4}$ During the first period of ischemia, there are essentially no changes in the levels of ATP, PCr, or Pi. Oxidative phosphorylation is still the major energy source for maintenance of myocardial homeostasis. During the second phase of ischemia (survival time), $\mathrm{PCr}$ is used to replenish ATP stores in the cytoplasm, which leads to a decrease in $\mathrm{PCr}$ level accompanied by a rise in Pi. This period ends when the $\mathrm{PCr}$ level decreases to approximately $40 \%$ of its normal value. In the present study 43 minutes of normothermic retrograde cardioplegia resulted in a decrease in $\mathrm{PCr}$ only to $53.5 \% \pm 27.3 \%$ of its control level. In addition, the final $\mathrm{pHi}$ at the end of 43 minutes of retrograde cardioplegia $(6.83 \pm 0.17)$ was also significantly higher than the level $(\mathrm{pHi}=6.2 \mathrm{pH}$ unit) considered indicative of severe ischemic injury. Therefore, the levels of both $\mathrm{PCr}$ and $\mathrm{pHi}$ suggest that ischemic changes during retrograde cardioplegia were very mild. Moreover, complete recovery of energy metabolism and contractile function during reperfusion further demonstrated that the retrograde cardioplegia-associated ischemic changes observed in this study were not severe. Nevertheless, the reduction in $\mathrm{PCr}$ and $\mathrm{pHi}$ is negative in regard to myocardial protection, particularly if the ischemic time is prolonged.

Because the piglet hearts used in this study were relatively small, it was not practical to use two surface coils to monitor myocardial energy metabolism in both ventricular walls separately. The present ${ }^{31} \mathrm{P}$ spectroscopy study used a coil surrounding the whole heart. The observed changes in energy metabolism and intracellular $\mathrm{pH}$ during retrograde cardioplegia and antegrade cardioplegia reflected the effects of the cardioplegic techniques on both ventricular walls. Because RV perfusion with retrograde cardioplegia was poor in most hearts, it is possible that the actual imbalance in energy metabolites and intracellular $\mathrm{pH}$ in the $\mathrm{LV}$ wall was less than that observed in the whole heart.

In this study the piglet hearts were perfused with modified Krebs-Henseleit solution, which is probably the major limitation of the present study. It has been demonstrated that blood-based cardioplegia offers several important advantages relative to crystalloid cardioplegia, including its higher oxygen-carrying capacity, higher buffer capacity, more appropriate osmotic load, endogenous oxygen-derived free radical scavengers, and the rheologic effects of red blood cells. ${ }^{23-25}$ The latter have been suggested to play an important role in the microcirculation. ${ }^{24,25}$ The effects of retrograde cardioplegia on myocardial perfusion and energy metabolism may have been improved if blood cardioplegia had been used in this study.

In addition, it should be mentioned that congenital heart defects in neonates and infants may be associated with pathologic changes in the RV, such as RV hypertrophy and pulmonary hypertension. These pathologic changes may compromise the efficacy of retrograde cardioplegia. Therefore, extrapolation of the results of this study into clinical situations should be done with caution.

In summary, under similar perfusion pressures, myocardial perfusion provided by retrograde cardioplegia is slightly less than that needed to sustain normal myocardial energy under normothermic conditions; however, it is sufficient for complete metabolic and functional recovery. The ability of retrograde cardioplegia to perfuse the RV myocardium is poor and varies between individuals.

We acknowledge the technical assistance of Mrs Lori Gregorash and Mrs Rachelle Mariash.

\section{REFERENCES}

1. Bove EL, Cooper J, Stark J. Cardioplegic protection of the child's heart. J Thorac Cardiovasc Surg 1984;88:287-93.

2. Fujiwara T, Heinle J, Britton L, Mayer JE Jr. Myocardial preservation in neonatal lambs: comparison of hypothermia with crystalloid and blood cardioplegia. J Thorac Cardiovasc Surg 1991;101:703-12.

3. Tchervenkov CI, Quantz M. Issues in newborn myocardial protection. In: Chiu RCJ, editor. Cardioplegia: current concepts and controversies. Austin: R.G. Landes Company; 1993. p. 52-64.

4. Young JN, Choy IO, Silva NK, Obayashi DY, Barkan HE. Antegrade cold blood cardioplegia is not demonstrably advantageous over cold crystalloid cardioplegia in surgery for congenital heart disease. J Thorac Cardiovasc Surg 1997;114:1002-8.

5. Gebhard MM, Bretschneider HJ, Schnabel PA. Cardioplegia principles and problems. In: Sperelakis N, editor. Physiology and 
pathophysiology of the heart. 3rd ed. Boston: Kluwer Academic; 1995. p. 731-43.

6. Rebeyka IM, Diaz RJ, Augustine JM, Herman SL, Wilson GJ, Coles JG, et al. Effect of rapid cooling contracture on ischemic tolerance in immature myocardium. Circulation 1991;84(Suppl 5):III-389-93.

7. Shirai T, Rao V, Weisel RD, Ikonomidis JS, Hayashida N, Ivanov $\mathrm{J}$, et al. Antegrade and retrograde cardioplegia: Alternate or simultaneous? J Thorac Cardiovasc Surg 1996;112:787-96.

8. Donnelly AJ, Djuric M. Cardioplegia solution. Am J Hosp Pharm 1991;48:2444-60.

9. Vaage J. Retrograde cardioplegia: when and how. Scand J Thorac Cardiovasc Surg 1993;41(Suppl):59-66.

10. Rao V, Cohen G, Weisel RD, Shiono N, Nonami Y, Carson SM, et al. Optimal flow rates for integrated cardioplegia. J Thorac Cardiovasc Surg 1998;115:226-35.

11. Gates RN, Laks H, Drinkwater DC Jr, Ardehali A, Aharon AS, Zaragoza AM, et al. Can improved microvascular perfusion be achieved by using both antegrade and retrograde cardioplegia? Ann Thorac Surg 1995;60:1308-11.

12. Ingwall JS. Phosphorus nuclear magnetic resonance spectroscopy of cardiac and skeletal muscles. Am J Physiol 1982;242:H72944.

13. Bottomley PA. MR spectroscopy of the human heart: the status and the challenges. Radiology 1994;191:593-612.

14. Hayashida N, Weisel RD, Shirai T, Ikonomidis JS, Ivanov J, Carson SM, et al. Tepid antegrade and retrograde cardioplegia. Ann Thorac Surg 1995;59:723-9.

15. Salerno TA, Christakis GT, Abel J, Houck J, Barrozo CAM, Fremes SE, et al. Techniques and pitfalls of retrograde continuous warm blood cardioplegia. Ann Thorac Surg 1991;51:1023-5.

16. Yau TM, Ikonomidis JS, Weisel RD, Mickle DA, Hayashida N,
Ivanov $\mathrm{J}$, et al. Which techniques of cardioplegia prevent ischemia? Ann Thorac Surg 1993;56:1020-8.

17. Menasche P. Retrograde perfusion. In: Piper HM, Preusse CJ, editors. Ischemia-reperfusion in cardiac surgery. London: Kluwer Academic; 1993. p. 255-66.

18. Ratajczyk-Pakalska E. The coronary venous anatomy. In: Meerbaum S, editor. Myocardial perfusion, reperfusion: coronary venous retroperfusion. New York: Springer-Verlag; 1990. p. 51-91.

19. Hammond GL, Moggio RA. Function of microvascular pathways in coronary circulation. Am J Physiol 1971;220:1463-7.

20. Esperanca-Pina JA, Correira M, O'Neill G. Morphological study on the thebesian veins of the right cavities of the heart in the dog. Acta Anat 1975;92:310-20.

21. Rudis E, Gates RN, Laks H, Drindwater DC, Ardehali A, Aharon A, et al. Coronary sinus ostial occlusion during retrograde delivery of cardioplegic solution significantly improves cardioplegic distribution and efficacy. $\mathrm{J}$ Thorac Cardiovasc Surg 1995;109:941-7.

22. Masuda M, Asou T, Kohno H, Nakamura Y, Morita S, Shiki K, et al. A comparative study of retrograde cardioplegic delivery via coronary sinus and right atrium. Rinsho Kyobu Geka 1987;7:40-4.

23. Weisel RD, Mickle DAG, Finkle CD. Delayed myocardial metabolic recovery after blood cardioplegia. Ann Thorac Surg 1989;48:503-7.

24. Suaudeau J, Schaffer B, Daggett WM. Role of procaine and washed red cells in the isolated dog heart perfused at $5^{\circ} \mathrm{C}$. J Thorac Cardiovasc Surg 1982;84:886-96.

25. Heitmiller RF, Deboer LWV, Geffin GA, Toal KW, Fallon JT, Drop LJ, et al. Myocardial recovery after hypothermic arrest: a comparison of oxygenated crystalloid to blood cardioplegia. Circulation 1985;64(Suppl):II-75-80. 